\title{
Segmentation of the Retinal Vasculature within Spectral-Domain Optical Coherence Tomography Volumes of Mice
}

\author{
Wenxiang Deng ${ }^{1}$, Bhavna Antony ${ }^{1}$, Elliott H. Sohn ${ }^{2}$, Michael D. Abràmoff ${ }^{2,1,3}$, \\ and Mona K. Garvin ${ }^{3,1}$ \\ 1 Electrical \& Computer Engineering, The University of Iowa, Iowa City, IA, USA \\ 2 Ophthalmology \& Visual Sciences, The University of Iowa, Iowa City, IA, USA \\ ${ }^{3}$ Iowa City VA Healthcare System, Iowa City, IA, USA
}

\begin{abstract}
Automated approaches for the segmentation of the retinal vessels are helpful for longitudinal studies of mice using spectral-domain optical coherence tomography (SD-OCT). In the SD-OCT volumes of human eyes, the retinal vasculature can be readily visualized by creating a projected average intensity image in the depth direction. The created projection images can then be segmented using standard approaches. However, in the SD-OCT volumes of mouse eyes, the creation of projection images from the entire volume typically results in very poor images of the vasculature. The purpose of this work is to present and evaluate three machine-learning approaches, namely baseline, single-projection, and all-layers approaches, for the automated segmentation of retinal vessels within SD-OCT volumes of mice. Twenty SD-OCT volumes $(400 \times$ $400 \times 1024$ voxels) from the right eyes of twenty mice were obtained using a Bioptigen SD-OCT machine (Morrisville, NC) to evaluate our methods. The area under the curve (AUC) for the receiver operating characteristic (ROC) curves of the all-layers approach, 0.93, was significantly larger than the AUC for the single-projection (0.91) and baseline (0.88) approach with $p<0.05$.
\end{abstract}

\section{Introduction}

With the increasing use of spectral-domain optical coherence tomography (SDOCT) in studies involving mice [1-4], there is a great need for automated image segmentation approaches. As a starting point, Antony et al. [5] as well as Srinivasan et al. [6] used automatic methods to segment surfaces in mouse SD-OCT volumes. Another structure of importance is the retinal vasculature. The automated segmentation of retinal vessels is important not only for the measurement of vasculature properties (such as vessel widths and tortuosity), but also to help with image registration and longitudinal studies. For example, in Srinivasan et al.'s approach [6], the location of vessels were used to remove artifacts in layer segmentations. In other studies, such as in the work of Gabriele et al. [2], vessel information was used to help with manual alignment to allow for better comparisons of longitudinal images.

X. Chen, M. K. Garvin, J. Liu, E. Trucco, Y. Xu (Eds.): OMIA 2015, Held in Conjunction with MICCAI 2015, Munich, Germany, Iowa Research Online, pp. 65-72, 2015. Available from: http://ir.uiowa.edu/omia/2015_Proceedings/2015/ 
With human data, the automated segmentation of retinal blood vessels in color fundus photographs is a well-understood problem and has many published papers [7-9]. Many of them can extend naturally to OCT projection images in human retinal studies. Approaches have also been presented for the segmentation of retinal vessels in SD-OCT volumes [10,11].

Segmenting vessels in SD-OCT data of mice needs different approaches than in the human case because of the differences between mice and human retinal images. More specifically, differences can be seen in example projection images in Fig. 1. Projection images for human (Fig. 1(a) and Fig. 1(b)) have much higher contrast between vessels and background than those of mice (Fig. 1(c) and Fig. 1(d)). However, for mouse SD-OCT images, very limited work has been presented for the segmentation of vessels. One implementation was briefly mentioned in a mouse retinal layer segmentation work by Srinivasan et al. [6], which served for correcting layer segmentations.

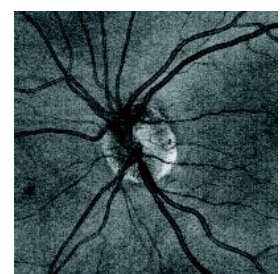

(a)

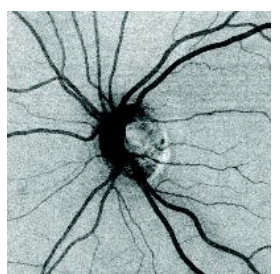

(b)

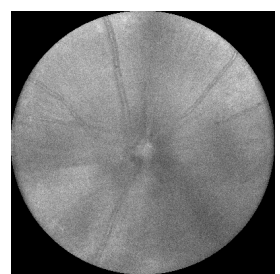

(c)

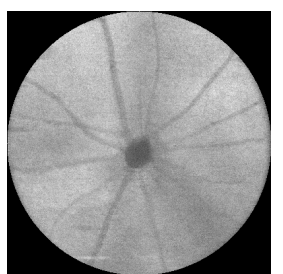

(d)

Fig. 1. Comparison SD-OCT projection images from a typical human and mouse. (a) Human: projection image from entire volume. (b) Human: projection image from segmented bottom layer. (c) Mouse: projection image from entire volume (notice the poor visibility of vessels). (d) Mouse: projection image from segmented bottom layer.

In this work, we propose three approaches for segmenting the mouse vasculature using a $k$-NN classifier. The first approach uses a similar approach as proposed by Niemeijer et al. [10] for human SD-OCT images involving a single layer for projection. The second classification approach involves a single projection of a set of layers and more initial features (reduced through feature selection). The last approach extracts features from projection images of all segmented retinal layers, instead of single projection image as in the first two. For convenience, we call these methods by baseline, single-projection, and all-layers approaches.

\section{Methods}

An overview of the three approaches we propose is illustrated in Fig. 2. Each approach consists of four steps. First, a total of eight retinal surfaces are segmented using the graph-theoretic approach proposed by Antony et al. [5] (Section 2.1). Second, 2D projection images are created using intraretinal layers from the segmented surfaces (Section 2.2). Third, a collection of features are generated from 


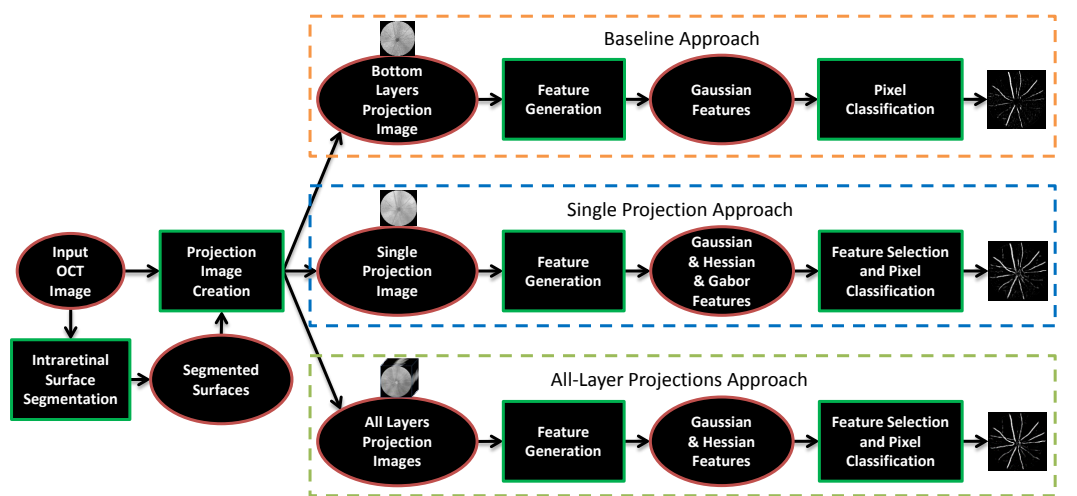

Fig. 2. Schematic overview of the three methods proposed.

projection images using Gaussian, Hessian, and Gabor-based filters. Each approach uses a different selection of features (Section 2.3). Finally, a forward feature selection is performed to obtain better feature sets (for the single-projection and all-layers approaches), and pixel classification is used to create a blood vessel probability map (Section 2.4).

\subsection{Intraretinal Layer Segmentation}

The layer segmentation is performed first using an established graph-theoretic method proposed by Antony et al. [5] to segment surfaces in each mouse SDOCT volume. Within the approach, multiple 3D surfaces in SD-OCT scans are segmented using the graph-theoretic approach $[12,13]$ that guarantees a global optimal solution with respect to the cost function. Using a similar approach to human-based approaches [13,14], the outer surfaces marked in red in Fig. 3(a) are simultaneously segmented first. Then the remaining surfaces, marked in yellow, are segmented. A multi-resolution approach [14] was also used in each of these steps in order to reduce computation time. The cost functions used here consisted of an on-surface cost term derived from gradient images computed using Gaussian derivative filters. Fig. 3(a) shows an example result of the layer segmentation.

\subsection{Projection Image Creation}

In SD-OCT volumes of humans, vessels are often still visible in the projection image obtained by averaging the whole volume (Fig. 1(a)). However, vessels are barely visible in similar projection images of mice data (Fig. 1(c)). Thus, in this work, all three proposed approaches use some type of layer-based projection image.

In the baseline approach, a projection image from the volume is created combining the inner segments (IS) to the retinal pigment epithelium (RPE) layers 
in accordance with the work of Niemeijer et al. [10]. For the single-projection approach, we create one overall $2 \mathrm{D}$ projection image using all of layers from the outer nuclear layer (ONL) to RPE. This combination of layers was experimentally chosen to create a single projection image with the best visually separable vessels. For the all-layers approach, we create 7 projection images (one for every segmented layer). Conceptually, we generate a $400 \times 400 \times 7$ volume instead of $400 \times 400 \times 1024$ to find blood vessels. Fig. 3 shows example of layers used and the corresponding projection images.

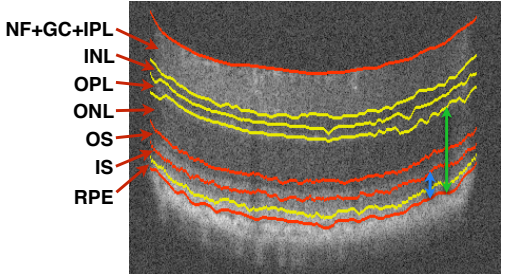

(a)

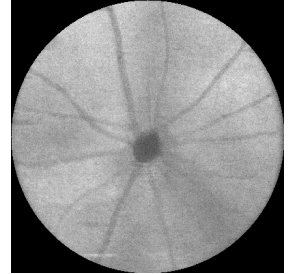

(b)

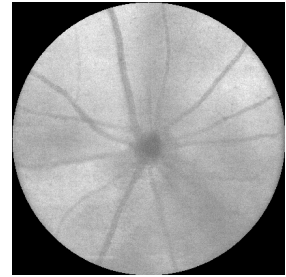

(c)
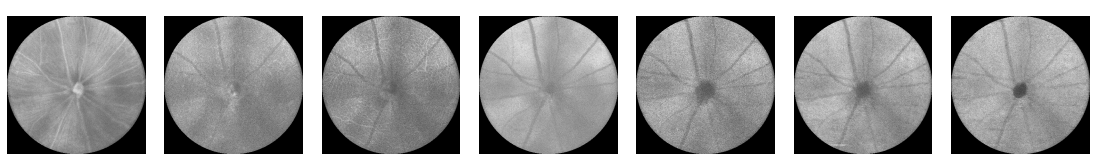

(d)

Fig. 3. Projection images for three approaches. (a) Layers used for creating projection images shown in single slice of $3 \mathrm{D}$ volume (blue arrow $=$ baseline approach; green arrow $=$ single-projection approach). (b) Projection image for baseline approach. (c) Projection image for single-projection approach. (d) Seven projection images for alllayers approach (one for each layer).

\section{$2.3 \quad$ Feature Generation}

The features extracted from each projection image are based on a combination of Gaussian, Hessian, and Gabor filters. Gaussian filters including Gaussian derivatives are commonly used to generate features and were also adopted in the human approach [10]. In particular, we applied Gaussian filters from 0 to $2^{\text {nd }}$ order derivatives $\left(L, L_{x}, L_{y}, L_{x y}, L_{x x}, L_{y y}\right)$ with scale $\sigma$ of $1,2,4,8,16$ to obtain a 30-dimension feature vector for each pixel of each projection image.

For Hessian-based features, a method based on point detection in [15] was used for creating filters. The filters were generated based on $2^{\text {nd }}$ order derivative Gaussian components $\left(L_{x y}, L_{x x}, L_{y y}\right)$ in Hessian matrix and angles of rotation. A collection of $\sigma(2,3,4,5)$ for Gaussian derivatives and rotations $\theta\left(0^{\circ}\right.$ to $180^{\circ}$ in $15^{\circ}$ increments) were used to generate a total of 48 features.

Gabor features can also be used for identifying vessels [16]. For Gabor features, filters with standard deviation $\sigma$ of $1,2,4,6$ and orientations $\theta$ from $0^{\circ}$ to 
$180^{\circ}$ using $15^{\circ}$ increments generated 48 Gabor-energy features. The mean and variance from symmetric Gabor filters were also used as features. In total, there were 144 Gabor features for each pixel of each projection image.

For each of the three approaches, we use different features. In the baseline method, only features generated using Gaussian filter banks are used to be consistent with [10]. In the single-projection method, we use all of the Gaussian, Hessian, and Gabor features mentioned above (222 features before feature selection). For the all-layers method, because including Gabor features would generate more than 1500 features in total, we only apply Gaussian and Hessian-based filters to generate $(30+48) \times 7=546$ features from 7 projection images. All features were normalized to have zero mean and unit variance.

\subsection{Feature Selection and Pixel Classification}

A sequential forward feature selection approach [17] is used as part of each crossvalidation training step to select a smaller and more helpful set of features for classification. (In the baseline approach, feature selection is not used because of the relatively small feature-set size and for consistency with [10].) As part of feature selection and the final classification, a $k$-NN classifier $(k=31$ as also in the human-based approach [10]) with soft labels is used for identifying pixels as vessel or non-vessel.

\section{Experimental Methods}

For validation of the method, we use 20 SD-OCT images $(400 \times 400 \times 1024$ voxels; $3.5 \times 3.5 \times 1.53 \mu \mathrm{m}$ per voxel) all from right eyes of 20 mice from a Bioptigen scanner. Using information from each of the layers as well as the overall best single projection image, pixels in the $2 \mathrm{D}$ projection images are manually labeled as vessel or non-vessel.

A cross-validation strategy is used to evaluate each approach. In particular, the 20 images are randomly divided into 5 sets with 4 images in each set. For each of the 5 sets, a training phase as detailed in the next paragraph is applied (also including feature selection) to the images in the remaining 16 images and this trained approach is applied to the 4 images in the set during the testing phase. Applying this training/testing process for each of the 5 sets results in the classified result for all 20 images.

The training phase for the single-projection and all-layers approaches includes feature selection (Section 2.4). In particular, the 16 images in the training phase are divided into two image subsets ( 8 in each) - a reference training subset and testing training subset - to find the best features. From the two training subsets, for speed performance, we randomly pick a subset of 100,000 training reference and 100,000 training testing instances to be used in the feature selection process. A total number of 20 features are selected.

For each of the five testing cross-validation rounds, the same 16 images in the training phase are used as the reference set, with 200,000 points being randomly 
selected. Then, every pixel point in the four images to be tested is classified as vessel or non-vessel using the $k$-NN classifier.

Receiver Operating Characteristic (ROC) curves are obtained by computing the sensitivity and specificity using different thresholds $(i=0,1, \ldots, k)$ of the soft-labeled images. The area under the curve (AUC) of the ROC curves are computed to evaluate the segmentation results. The AUC of approaches are then compared using the bootstrapping method by Carpenter and Bithell [18] using the pROC package in $\mathrm{R}[19]$.

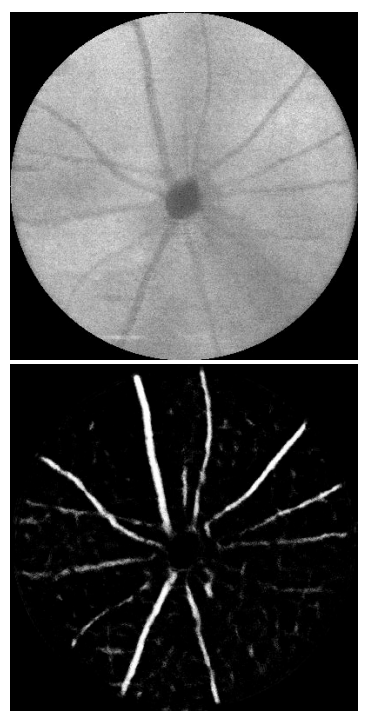

(a)
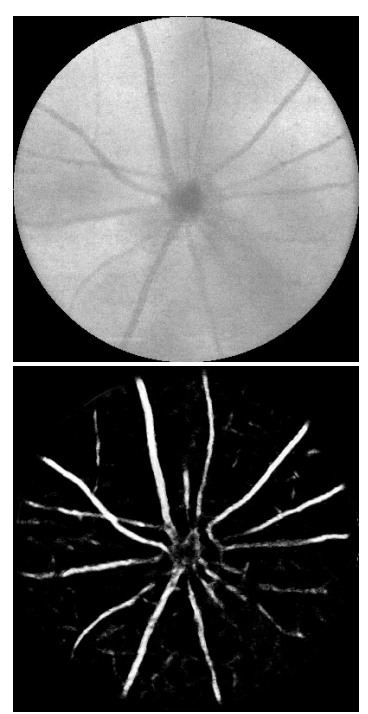

(b)
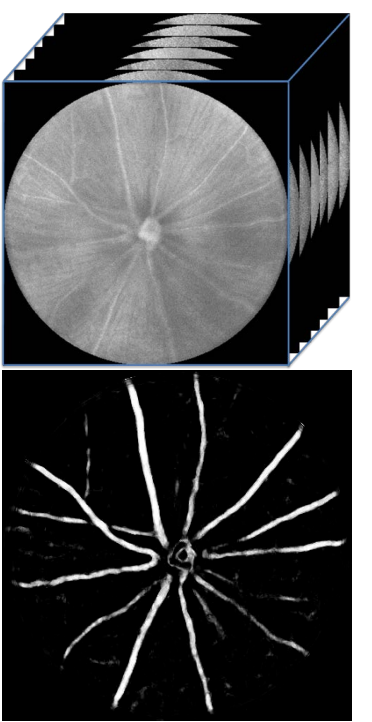

(c)

Fig. 4. Example projection image(s) and result from the (a) baseline approach, (b) single-projection approach and (c) all-layers approach.

\section{Results}

Example results can be seen in Fig. 4. The all-layers approach generates the best overall vessel image. It has cleaner background, and segmented blood vessels are clear and continuous. For the baseline approach, not only are more non-vessels segmented as vessels, some vessels cannot be successfully segmented compared with the other two approaches.

ROC curves of all three methods are plotted in Fig. 5. As shown in Fig. 5, the AUC of the ROC curve for the baseline approach is 0.88 . The AUC for the single-projection approach is 0.91 , and is significantly better than the baseline approach $(p<0.05)$. The AUC for the all-layers approach is 0.93 , which is significantly better than that of the other two approaches $(p<0.05)$. 


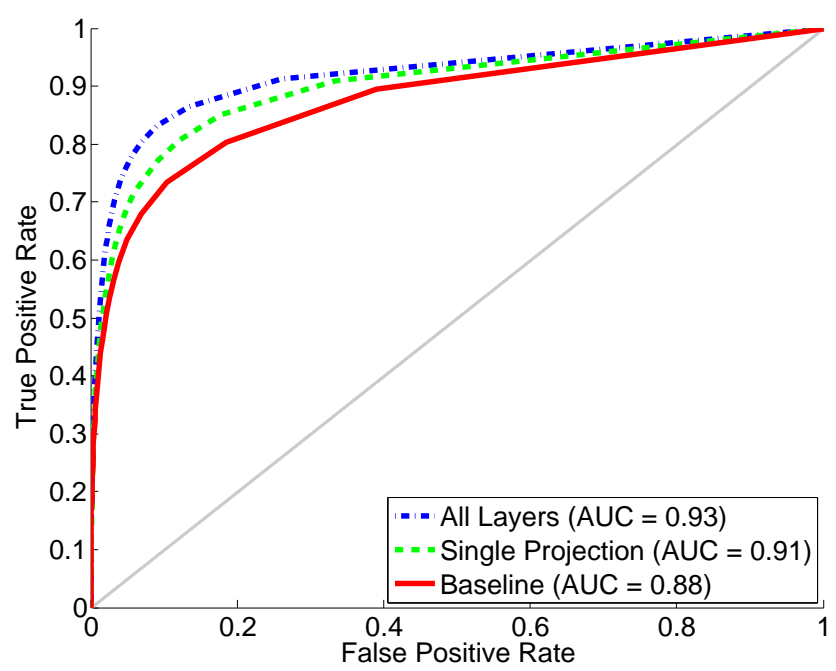

Fig. 5. ROC curves for baseline, single-projection, and all-layers approach.

\section{Conclusion}

There are three approaches proposed in this work for segmenting retinal vessels in SD-OCT volumes of mice. Our results show that with baseline approach adopted from prior human-based work, some of the vessels cannot be successfully segmented. Using the other two new approaches results in a significant improvement, with the method using projection images from all layers providing the best overall results with more visible vessels and higher contrast. Having such automated retinal vessel segmentation approaches as presented will greatly enhance the image-analysis possibilities of longitudinal SD-OCT volumes of mice.

Acknowledgements. This work was supported, in part, by the Department of Veterans Affairs Career Development Award 1IK2RX000728.

\section{References}

1. Huber, G., Beck, S.C., Grimm, C., Sahaboglu-Tekgoz, A., Paquet-Durand, F., Wenzel, A., Humphries, P., Redmond, T.M., Seeliger, M.W., Fischer, M.D.: Spectral domain optical coherence tomography in mouse models of retinal degeneration. Invest Ophthalmol Vis Sci 50(12) (2009) 5888-5895

2. Gabriele, M.L., Ishikawa, H., Schuman, J.S., Ling, Y., Bilonick, R.A., Kim, J.S., Kagemann, L., Wollstein, G.: Optic nerve crush mice followed longitudinally with spectral domain optical coherence tomography. Invest Ophthalmol Vis Sci 52(5) (2011) 2250-2254

3. Pennesi, M.E., Michaels, K.V., Magee, S.S., Maricle, A., Davin, S.P., Garg, A.K., Gale, M.J., Tu, D.C., Wen, Y., Erker, L.R., et al.: Long-term characterization of 
retinal degeneration in $\mathrm{rd} 1$ and $\mathrm{rd} 10$ mice using spectral domain optical coherence tomography. Invest Ophthalmol Vis Sci 53(8) (2012) 4644-4656

4. Puk, O., de Angelis, M.H., Graw, J.: Longitudinal fundus and retinal studies with SD-OCT: a comparison of five mouse inbred strains. Mamm Genome 24(5-6) (2013) 198-205

5. Antony, B.J., Jeong, W., Abràmoff, M.D., Vance, J., Sohn, E.H., Garvin, M.K.: Automated 3D segmentation of intraretinal surfaces in SD-OCT volumes in normal and diabetic mice. Transl Vis Sci Technol 3(5) (2014)

6. Srinivasan, P.P., Heflin, S.J., Izatt, J.A., Arshavsky, V.Y., Farsiu, S.: Automatic segmentation of up to ten layer boundaries in SD-OCT images of the mouse retina with and without missing layers due to pathology. Biomed Opt Express 5(2) (2014) 348-365

7. Zana, F., Klein, J.C.: Segmentation of vessel-like patterns using mathematical morphology and curvature evaluation. IEEE Trans Image Process 10(7) (2001) 1010-1019

8. Niemeijer, M., Staal, J., van Ginneken, B., Loog, M., Abramoff, M.D.: Comparative study of retinal vessel segmentation methods on a new publicly available database. In: SPIE Medical Imaging. Volume 5370. (2004) 648-656

9. Soares, J.V., Leandro, J.J., Cesar, R.M., Jelinek, H.F., Cree, M.J.: Retinal vessel segmentation using the 2-D Gabor wavelet and supervised classification. IEEE Trans Med Imag 25(9) (2006) 1214-1222

10. Niemeijer, M., Garvin, M.K., van Ginneken, B., Sonka, M., Abràmoff, M.D.: Vessel segmentation in 3D spectral OCT scans of the retina. In: SPIE Medical Imaging. Volume 6914. (2008) 69141R (8 pages)

11. Xu, J., Tolliver, D., Ishikawa, H., Wollstein, G., Schuman, J.S.: 3D OCT retinal vessel segmentation based on boosting learning. In: World Congress on Medical Physics and Biomedical Engineering, Springer (2009) 179-182

12. Li, K., Wu, X., Chen, D.Z., Sonka, M.: Optimal surface segmentation in volumetric images-a graph-theoretic approach. IEEE Trans Pattern Anal Machine Intell 28(1) (2006) 119-134

13. Garvin, M.K., Abràmoff, M.D., Wu, X., Russell, S.R., Burns, T.L., Sonka, M.: Automated 3-D intraretinal layer segmentation of macular spectral-domain optical coherence tomography images. IEEE Trans Med Imag 28(9) (2009) 1436-1447

14. Lee, K., Niemeijer, M., Garvin, M.K., Kwon, Y.H., Sonka, M., Abràmoff, M.D.: 3-D segmentation of the rim and cup in spectral-domain optical coherence tomography volumes of the optic nerve head. In: SPIE Medical Imaging. Volume 7262. (2009) $72622 \mathrm{D}$ (9 pages)

15. Klein, A., Renema, W.K., Oostveen, L.J., Kool, L.J.S., Slump, C.H.: A segmentation method for stentgrafts in the abdominal aorta from ECG-gated CTA data. In: SPIE Medical Imaging. Volume 6916. (2008) 69160R (10 pages)

16. Bhuiyan, A., Nath, B., Chua, J., Kotagiri, R.: Blood vessel segmentation from color retinal images using unsupervised texture classification. In: Image Processing, 2007. ICIP 2007. IEEE International Conference on. Volume 5., IEEE (2007) V-521

17. Whitney, A.W.: A direct method of nonparametric measurement selection. IEEE Comput 100(9) (1971) 1100-1103

18. Carpenter, J., Bithell, J.: Bootstrap confidence intervals: when, which, what? A practical guide for medical statisticians. Stat Med 19(9) (2000) 1141-1164

19. Robin, X., Turck, N., Hainard, A., Tiberti, N., Lisacek, F., Sanchez, J.C., Müller, M.: pROC: an open-source package for $\mathrm{R}$ and $\mathrm{S}+$ to analyze and compare ROC curves. BMC Bioinformatics 12(1) (2011) 77 